\section{Uma proposta de criação de um sistema para monitoramento dos casos de mesotelioma maligno em Curitiba, Paraná, Brasil}

\author{
A proposal for the creation of a system to \\ monitor cases of malignant mesothelioma \\ in Curitiba, Paraná, Brazil
}

\author{
Una propuesta de creación de un sistema para \\ la supervisión de los casos de mesotelioma \\ maligno en Curitiba, Paraná, Brasil
}

\author{
Luciana Puchalski Kalinke 1 \\ Marco Aurélio Kalinke 1,2 \\ Leila Maria Mansano Sarquis 1 \\ Larissa Marcondes 1 \\ Tatiana Halfeld 1 \\ Carolina Mensi 3 \\ Dario Consonni 3
}

doi: $10.1590 / 0102-311 \times 00171917$

\section{Resumo}

Este estudo objetivou propor a criação de um sistema de monitoramento dos casos de mesotelioma maligno no Município de Curitiba, Paraná, Brasil, desenvolvido com base no modelo italiano. Trata-se de uma pesquisa-ação tipo diagnóstica, que utiliza as fases exploratória e de planejamento, desenvolvida no período de julho de 2015 a maio de 2017. Utilizaram-se como ferramentas de busca os seguintes instrumentos: Integrador de Registro Hospitalar de Câncer, com as morfologias específicas para mesotelioma; Registro Hospitalar de Câncer, com os códigos C38.4 e C45, da Classificação Internacional de Doenças, 10 a revisão, elou registros codificados pelo CID-O, com as topografias C38 e C48; Registro de Câncer de base populacional da Secretaria Municipal de Saúde de Curitiba, com os mesmos códigos. Também foram conhecidos, analisados e adaptados para a realidade brasileira o modelo, os questionários e o software de registro de mesotelioma da Lombardia, na Itália. Observou-se que, no Integrador de Registro Hospitalar de Câncer, foram registrados $15 \mathrm{ca}$ sos de mesotelioma. No Registro Hospitalar de Câncer do hospital universitário, foram dois. No hospital oncológico, 16. Os dados do Registro de Câncer de Base Populacional, por sua vez, indicaram 317 registros relativos ao periodo. Apesar de algumas informações estarem mais completas, existe a falta de dados relacionados à história laboral, impossibilitando estabelecer o nexo causal. Com o aumento do número de casos de mesotelioma previstos para as próximas décadas e o atendimento às demandas legais, a implantação de registros torna-se essencial para auxiliar no conhecimento, no acompanhamento, na determinação de nexo causal e nas fontes de contaminação específicas no país.

\section{Correspondência}

M. A. Kalinke

Universidade Tecnológica Federal do Paraná.

Av. Sete de Setembro 3165, Curitiba, PR 80230-901, Brasil.

marcokalinke@yahoo.com.br

\section{Universidade Federal do Paraná, Curitiba, Brasil.}

2 Universidade Tecnológica Federal do Paraná, Curitiba, Brasil.

3 Fondazione IRCCS Ca' Granda - Ospedale Maggiore

Policlinico, Milano, Italia. 


\section{Introdução}

O mesotelioma maligno é um tipo de câncer raro, com alto grau de malignidade, disseminação e um tempo de latência que pode chegar a mais de quarenta anos ${ }^{1}$. Desenvolve-se a partir da mutação de células mesoteliais que revestem a superfície da pleura, do peritônio, do pericárdio e da túnica vaginalis. Está classificado entre os tumores pleurais primários em $90 \%$ dos cânceres de pleura 2.

Devido às semelhanças com adenocarcinoma de outros órgãos, seu diagnóstico é difícil e com baixa precisão, e 10\% dos casos são confirmados somente nas autópsias 3 . Além da incidência crescente de mesotelioma maligno 4 , nota-se que pacientes com essa doença têm mau prognóstico e, embora tratamentos como cirurgia e quimioterapia, principalmente com anticorpos monocloidais, sejam parcialmente bem-sucedidos, com relação ao prolongamento e à melhoria da qualidade de vida, a cura não está disponível 5. Segundo as estimativas da Organização Mundial da Saúde (OMS), mais de 181 mil pessoas morrem anualmente de mesotelioma, asbestose e câncer de pulmão, resultantes de exposições ocupacionais relacionadas ao amianto 6 .

Existe uma associação direta entre o surgimento dos mesoteliomas e exposição ao amianto 7 . O fator ocupacional é responsável por cerca de 80 a $85 \%$ dos casos, com prevalência no sexo masculino, por representar a maior parcela de trabalhadores em atividades que utilizam o amianto como matéria-prima ${ }^{8}$. Nos casos de exposição ambiental (4,2\%) e não ocupacional (1,6\%) 9, a presença é maior entre as mulheres, o que está possivelmente relacionado ao fato de serem moradoras de áreas vizinhas às fábricas e por conta da limpeza dos uniformes dos familiares.

O amianto é um mineral de origem rochosa, composto por fibras divididas em dois grandes grupos: anfibólios e serpentinas (crisotila ou amianto branco). É utilizado como isolante acústico e térmico, qualidades que despertaram seu interesse para uso industrial10. Cerca de 125 milhões de pessoas no mundo estão expostas a esse mineral no local de trabalho, e uma em cada três mortes por câncer ocupacional tem causa no amianto 11.

No Brasil, espera-se um aumento da incidência dos óbitos por mesotelioma maligno e por câncer de pleura para a próxima década, particularmente entre os anos de 2021-2026, aproximadamente trinta anos após o pico de consumo do amianto no país 12. No entanto, os dados para calcular essa incidência são frágeis. Goiânia, Goiás, na região Centro-oeste (onde fica a maior mina exploradora brasileira de amianto), apresentou, no período de 2001 a 2005, taxas de 0,14/100 mil entre os homens e de 0,23/100 mil entre as mulheres 13 .

Na direção oposta de países como Suíça, França, Itália, entre outros, que aboliram o amianto e seus derivados, o Brasil baniu na década de 1990 apenas os anfibólios, porém legitimou a extração e a comercialização do crisótilo, com a Lei Federal no 9.055, de 1o de junho de 1995 14. Apenas em 2017, por decisão do Supremo Tribunal Federal (STF), o amianto foi definitivamente proibido no país, quando ainda existiam 47 empreendimentos autorizados a utilizá-lo, empregando um total de 4.938 trabalhadores 8 .

Em Curitiba (Paraná), a proibição do uso do crisótilo foi definida pela Lei Municipal no 14.172 15, vigente a partir de 7 de dezembro de 2015, que tratou também de regras para construções novas, reformas e notificação de casos. A lei estabelece que as doenças e óbitos relacionados à exposição ao amianto devem ser registrados no Sistema de Informações e Agravos de Notificação (SINAN) e acompanhados pelo Sistema Único de Saúde (SUS).

Se for considerado o fato de que em 2017 o Brasil era o terceiro maior produtor, consumidor e exportador mundial de crisótilo, ao comparar as taxas de incidência de mesotelioma maligno no país diante das taxas mundiais, observa-se discrepância de dados, indicando que possivelmente as informações relacionadas à quantidade de casos de mesotelioma maligno e de óbitos no Brasil são errôneas 16 . Ademais, a relação de casos com a história ocupacional dos pacientes é quase inexistente.

No Brasil, o percentual médio de ausência de informação sobre ocupação e história ocupacional para todas as topografias de câncer é de $40 \%$ para homens e de $52 \%$ para mulheres 17 . No estudo realizado pelo Instituto Nacional de Câncer José Alencar Gomes da Silva (INCA), que analisa o Sistema de Informação de Registro Hospitalar de Câncer (SisRHC) de 21 estados brasileiros, foi apontado que o percentual médio de ausência de informação sobre "ocupação" no registro é de $46 \% 18$.

Estudos brasileiros 10,12 têm se dedicado a discutir as questões do amianto e sua relação com as doenças ocupacionais. Porém, há falhas nos registros de pacientes, como falta de dados relativos a 
história ocupacional e a sua possível relação com a doença. Quando os dados estão disponíveis, em muitos casos são da ocupação atual ou da última ocupação referida pelo paciente. Entretanto, devido ao longo período de latência de alguns cânceres, como no caso do mesotelioma maligno, esses dados não são suficientes para estabelecer um nexo causal.

É nesse contexto que se apresenta o objetivo deste estudo, qual seja, de propor a criação de um sistema de monitoramento de casos de mesotelioma maligno no Município de Curitiba, desenvolvido com base no modelo italiano. Ele poderá auxiliar no acompanhamento dos casos, no conhecimento da história ocupacional, familiar e residencial (para verificação de eventual exposição anterior ao amianto) e no estabelecimento da relação do nexo causal entre o mesotelioma maligno e o amianto.

\section{Metodologia}

Para atingir os objetivos deste estudo, optou-se por seguir de forma adaptada os passos propostos pela pesquisa-ação tipo diagnóstica, utilizando-se as fases exploratória e de planejamento, no período de julho de 2015 a maio de 2017.

A fase exploratória foi dividida em três etapas e teve como objetivo verificar a problemática dos registros de casos de mesotelioma maligno na região de Curitiba, para conhecer como eles são realizados, a completude das suas informações e a disponibilização de dados para investigação do nexo causal. A temporalidade de busca no Integrador de Registro Hospitalar de Câncer (IntegradorRHC) do INCA, Registro Hospitalar de Câncer (RHC) de dois hospitais de referência de Curitiba (um universitário e outro oncológico) e no Registro de Câncer de Base Populacional (RCBP) da Secretaria Municipal de Saúde de Curitiba (SMS-Curitiba) está relacionada à disponibilidade dos dados disponíveis por completo.

Na primeira etapa, foi realizada a busca no IntegradorRHC do INCA pelas morfologias de mesotelioma maligno (9053/3: bifásica; 9052/3: epitelioide; 9051/3: fibroso e 9050/3: não especificado) com dados disponíveis de janeiro de 2001 a dezembro de 2014. Na segunda, foi realizada a busca dos casos de mesotelioma maligno no RHC dos dois hospitais de referência de Curitiba, com dados disponíveis entre 1995 e 2015, nos códigos C38.4 e C45, de acordo com a Classificação Internacional de Doenças 10a Revisão (CID-10), e suas derivações, e/ou registros codificados pelo CID-O com as topografias C38 e C48. Na terceira, foi analisado o RCBP da SMS-C, no período de 1998 a 2012, com os mesmos CID.

A segunda fase da pesquisa, relativa ao planejamento de um registro específico de casos de mesotelioma para o Brasil, tendo como piloto a cidade de Curitiba, objetivou conhecer, analisar e adaptar para a realidade brasileira os questionários e o software de registro utilizados na região da Lombardia, na Itália, bem como acompanhar in loco os procedimentos de registro de casos naquela região.

O Registro Nacional de Casos de Mesotelioma (Registro Nazionale dei Mesoteliomi - Renam) da região da Lombardia, é situado na Clinica del Lavoro de Milão, instituição legalmente responsável por recolher os dados dos casos de mesotelioma maligno desde 1o de janeiro de 2000. Entre seus objetivos, destacam-se: (a) revelar a incidência e a distribuição territorial de mesotelioma; (b) verificar a exposição anterior ao amianto; (c) reconhecer as fontes desconhecidas de exposição, promover projetos de pesquisa relacionados ao mesotelioma e exposição ao amianto; e (d) fornecer assistência médica e legal aos pacientes e seus familiares.

$\mathrm{Na}$ fase de planejamento, também foi desenvolvida uma proposta para a implantação de um registro piloto para a cidade de Curitiba, levando em consideração o cenário atual e a experiência do registro italiano.

Em relação aos aspectos éticos, esta pesquisa faz parte do projeto temático intitulado "O Adoecimento do Trabalhador e as Neoplasias Brônquio Pulmonares", que teve início em 2014 com uma parceria firmada entre a Universidade Federal do Paraná (UFPR), a Secretaria Estadual de Saúde do Paraná e a Clinica del Lavoro de Milão. A pesquisa recebeu financiamento da Coordenação de Aperfeiçoamento de Pessoal de Nível Superior (Capes), por meio do edital Professor Visitante Exterior - auxílio no 189571, e aprovação pelos Comitês de Ética em Pesquisa (CEP) do Setor de Ciências da Saúde da UFPR (parecer no 1.959.247), do CEP da SMS-C (parecer no 2.027.730) e do CEP do Hospital do Tra- 
balhador/Secretaria Estadual de Saúde, com a coparticipação do Hospital Erasto Gaertner (parecer no 2.035.991).

\section{Resultados}

\section{Registro de casos de mesotelioma maligno em Curitiba}

Na fase exploratória, em relação aos resultados da busca realizada nos registros citados, observou-se que no IntegradorRHC foram registrados 15 casos de mesotelioma maligno no Paraná, entre janeiro de 2001 e dezembro de 2014. Desses, em nove casos a morfologia era de tipo não especificada, quatro eram epitelioides, dois fibrosos e nenhum bifásico ${ }^{19}$. As informações relacionadas às atividades ocupacionais foram encontradas em $50 \%$ dos casos, sendo referentes apenas aos últimos três meses de atividade laboral.

O serviço do RHC do hospital universitário teve início em 2003, começando efetivamente a coleta de informações apenas no ano de 2004. Desse período até o momento da busca, foram registrados dois casos de mesotelioma maligno, nos anos de 2009 e 2015, referentes a pacientes da região metropolitana e de Curitiba. No RHC do hospital oncológico, o registro é mais antigo e completo, contendo informações que remontam ao início da década de 1990. Nesse período, foram encontrados 16 casos de mesotelioma maligno em pacientes de Curitiba, região metropolitana e interior do estado. Apesar das informações serem mais completas, existe a falta de dados relacionados à história laboral, impossibilitando estabelecer um nexo causal.

Os dados do RCBP indicam um total de 317 registros de casos notificados com origem topográfica C38 (coração, mediastino e pleura) e C48 (retroperitônio e peritônio). Desses casos, 20 eram mesotelioma maligno e 36 eram câncer de pleura, relativos ao período compreendido entre os anos de $1998 \mathrm{e}$ 2012. Destes, 33 eram de crianças e adolescentes menores de 18 anos. Não há, também neste registro, dados relativos à atividade laboral.

\section{Registro de mesoteliomas da região da Lombardia, na Itália}

A Itália foi um grande produtor e usuário de amianto no final da década de 1980, tendo banido o uso por completo em 1992. O principal fator que contribuiu para a criação do registro no país foi a determinação legal 20 de que todos os casos deveriam ser notificados.

Atualmente o setor de registro da Clinica del Lavoro de Milão é composto por uma equipe formada por registradores, que são assistentes administrativo e bolsistas. Todos os casos de mesotelioma são registrados, e o nexo causal é estabelecido em cerca de 95\% deles. A busca dos casos é realizada nas instituições de saúde em toda a região (cerca de 120), sendo obrigatória a comunicação de todos os casos suspeitos ou confirmados de mesotelioma pelos serviços de anatomia patológica, com envio de fax ou e-mail ao registro (Figura 1). Complementarmente, e como forma de controle, é solicitado aos serviços o envio de um relatório com todos os casos analisados, duas vezes ao ano.

Para cada caso notificado, os profissionais da clínica fazem a reconstrução detalhada da história médica do paciente, mediante a documentação de anamnese, exames diagnósticos (raio X e/ou tomografia), descrição da biópsia (toracoscopia, biópsia por agulha, intervenção cirúrgica), exame citológico e/ou histológico, imuno-histoquímica e eventual follow-up, para confirmar o diagnóstico. Caso a documentação clínica seja insuficiente, o registro solicita o prontuário do paciente e a colaboração do médico do primeiro atendimento. Com o diagnóstico clínico de mesotelioma confirmado, é feita a reconstrução de possível exposição ao amianto, com a aplicação de um instrumento específico, denominado Questionario Sulla Storia Di Lavoro e Sulle Abitudini Di Vita, com referência à história de trabalho e a hábitos de vida do paciente.

O questionário é aplicado por um profissional especializado em saúde ocupacional e contém perguntas relacionadas a dados pessoais, hábitos de fumo, levantamento das histórias profissional e extraocupacional, serviço militar, residencial e saúde. No tópico sobre história ocupacional, podese fazer a busca por ex-colegas de trabalho, na tentativa de esclarecer e identificar detalhadamente informações relativas a produtos, máquinas e/ou equipamentos de manuseio na empresa em que 


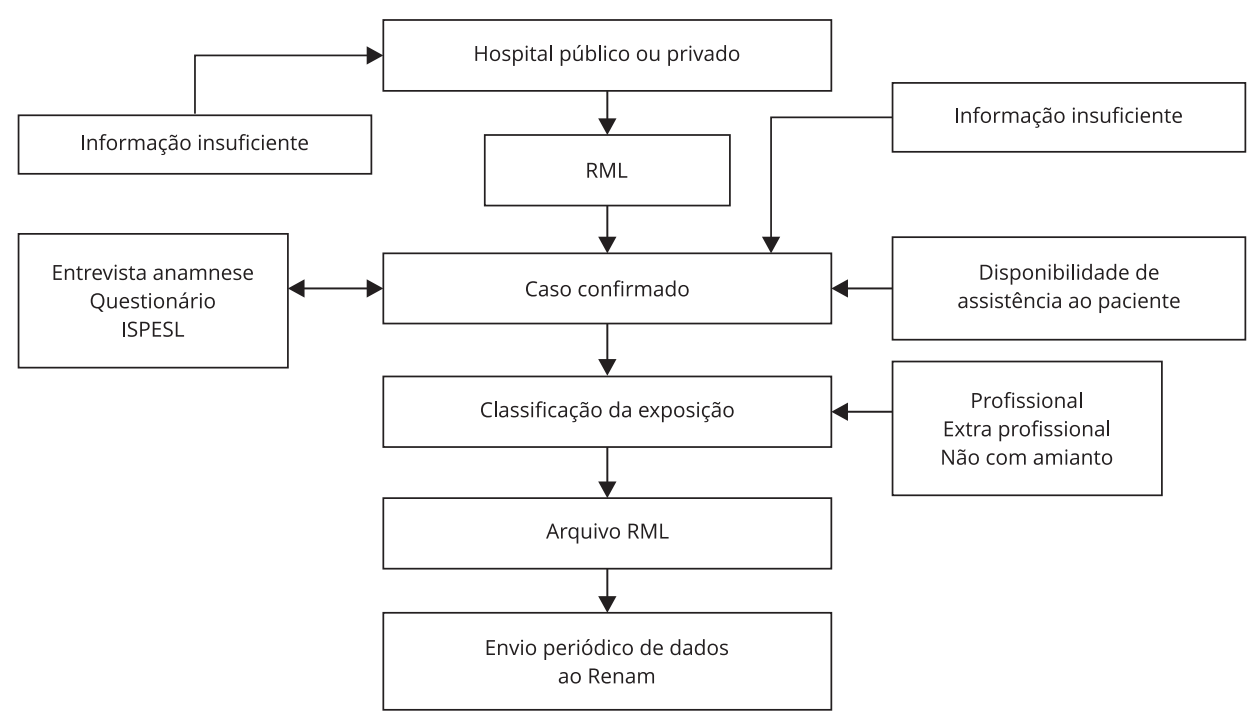

RML: Radionuclide Metrology Laboratory; ISPESL: Italian Institute for Occupational Safety and Prevention; Renam: Registro Nacional (Italiano) de casos de mesotelioma (Registro Nazionale dei Mesoteliomi). Nota: fluxo referente ao serviço italiano de investigação de casos de mesotelioma maligno.

trabalhavam, avaliando a existência ou não de amianto. Esse procedimento objetiva adquirir maior quantidade e melhor qualidade das informações.

Em um segundo momento, é realizada a avaliação dos casos por uma equipe multidisciplinar, que se reúne semanalmente para a discussão. A equipe é composta por especialistas em medicina do trabalho, oncologia, pneumologia, epidemiologia, higiene e tecnologia industrial. É importante destacar que a relação e a confirmação da exposição de amianto acontecem somente quando o diagnóstico clínico de mesotelioma é confirmado, evitando que informações conhecidas sobre a exposição pregressa influenciem no diagnóstico. Durante a discussão da equipe multidisciplinar, ocorre a classificação dos casos em relação ao tipo de exposição ao amianto (Quadro 1).

Com a finalização da investigação e sua devida classificação, os casos são registrados em um software dedicado ao armazenamento, à gestão das informações e à transmissão padronizada por todos os centros operacionais regionais ao Renam, sediado em Roma, Itália. Ele possibilita gerar relatórios que fornecem um panorama dos casos, classificações, tipos de mesotelioma, relação conhecida ou não com o amianto, entre outros.

A versão atual do software é a 2.0. Essa versão tem, entre suas funcionalidades, as particularidades de atribuir automaticamente o número do código identificativo de cada caso, auxiliar na identificação dos códigos atribuídos às diversas atividades produtivas e tarefas e, além disso, limitar os erros nas digitações das informações. Os dados são criptografados e separados em dois arquivos para serem enviados ao registro nacional, garantindo o sigilo das informações de cada um dos casos registrados. O software também pode fornecer taxas brutas e padronizadas de mesotelioma (total e para cada sede) por ano, sexo, faixa etária e município de residência, segundo o momento do diagnóstico. Para isso, são utilizados os arquivos da população residente por ano, sexo, faixa etária e município (disponíveis na página de internet do ISTAT - Istituto Nazionale di Statistica - https://www.istat.it/, equivalente ao IBGE).

Outro ponto importante considerado pelo registro da Clinica del Lavoro de Milão é a qualidade do levantamento de dados, que é garantida a partir de buscas ativas e periódicas em banco de dados 


\section{Quadro 1}

Classificação dos casos de mesotelioma em relação ao tipo de exposição ao amianto.

\begin{tabular}{|c|c|}
\hline Classificação dos casos & Tipo de exposição ao amianto \\
\hline Certa & Uso/exposição, com comprovação \\
\hline Provável & Uso/exposição, sem comprovação \\
\hline Possível & Atividade com amianto, sem manipulação direta \\
\hline Formas de exposição & Tipo de exposição ao amianto \\
\hline Familiar & Não exposto profissionalmente, mas conviveu com ao menos um trabalhador com exposição certa ou provável \\
\hline Ambiental & Viveu nas imediações de zonas industriais que utilizavam amianto \\
\hline Extraprofissional & Exposto ao amianto em atividades no ambiente doméstico ou em tempo livre \\
\hline Improvável & $\begin{array}{l}\text { São disponíveis informações de boa qualidade sobre atividade laboral e de vida, que indicam exposição a níveis } \\
\text { ambientais naturais de amianto }\end{array}$ \\
\hline Desconhecida & Quando não há evidência de exposição \\
\hline A definir & Está em curso o levantamento de informações para a avaliação da exposição \\
\hline Não classificável & Quando há incompletude e insuficiência das informações levantadas \\
\hline
\end{tabular}

Nota: classificação de exposição ao amianto adotada pelo serviço italiano de investigação de casos de mesotelioma maligno.

diferentes, tais como os serviços de anatomia patológica, registros de alta hospitalar de casos de câncer pleural, atestados de óbito com CID de mesotelioma, além da busca de casos com exposição profissional denunciadas ao Instituto de Seguridade Nacional. Dessa forma, ele consegue atingir o percentual máximo de registros de casos na região da Lombardia.

\section{Implantação de um registro de casos de mesotelioma}

A proposta para implantação de um Registro de Casos de Mesotelioma no Município de Curitiba é adaptada do modelo italiano, levando em consideração as especificidades brasileiras. Ele auxiliará tanto no conhecimento do número de casos para a comunidade e sua apresentação uniforme e real, quanto no atendimento às questões legais do Município de Curitiba. A Lei Municipal $n^{\circ} 14.17215$ dispõe de pontos importantes sobre o uso do amianto na cidade, proibindo a utilização de materiais ou artefatos que contenham quaisquer tipos de amianto ou asbesto, ou até mesmo outros minerais que acidentalmente tenham fibras de amianto na sua composição. Também destaca a necessidade de compreender a dimensão que o mesotelioma maligno atinge no município, conhecendo o número de casos totais, número de óbitos relacionados à doença e a sua relação com atividades laborais nas quais o amianto esteja presente.

Ancorados nos aspectos descritos anteriormente, é possível verificar a importância e a necessidade da implantação de um registro para o conhecimento, acompanhamento e controle dos casos de mesotelioma maligno em Curitiba. Secretarias de epidemiologia e de saúde do município, bem como hospitais de referência em oncologia ou na saúde do trabalhador são os locais adequados para sua implantação e desenvolvimento. Nas secretarias, a centralidade, abrangência e possibilidade de maior acompanhamento das notificações são aspectos relevantes. Já os hospitais, por se tratarem de centros de referência, possuem equipe especializada, com mais possibilidades para reuniões de discussão dos casos. No entanto, esse é um processo que se apresenta como longo e contínuo, no qual em diversos momentos os envolvidos deverão trabalhar em conjunto.

Além do local, é importante que o registro tenha recursos humanos disponíveis, como registradores e assistentes administrativos. Deve haver também uma equipe multiprofissional especializada em 
oncologia, medicina e segurança do trabalho, que deverá dar suporte para a realização das reuniões e discussão dos casos e para a busca da relação do nexo causal com o uso de amianto.

Independentemente do local físico em que se armazene o registro e dos recursos humanos disponíveis, é necessário que se faça cumprir o $\$ 1$ o do Art. 60 da Lei Municipal sobre a obrigatoriedade da notificação dos casos de mesotelioma. Dessa forma, entre as ações necessárias, merece destaque a parceria com os laboratórios de anatomia patológica. Os casos cujos resultados sejam de mesotelioma, confirmados ou suspeitos, deverão ser notificados com o nome do paciente, número do registro e nome da instituição, para que se faça a busca ativa dos demais dados. Outras instituições de saúde, tais como hospitais, clínicas e unidades de saúde também deverão notificar os casos confirmados ou suspeitos, deixando à disposição do registro toda documentação clínica e radiológica, para que seja possível levantar o seu histórico.

O registrador, ao portar toda a documentação clínica do paciente e após realizar um levantamento da história clínica, deverá encontrar o paciente ou um familiar próximo para realizar uma entrevista, durante a qual utilizará como apoio o instrumento denominado Questionário sobre a História de Trabatho e Hábitos de Vida versão 1.0 português - Brasil, que foi traduzido do italiano e adaptado transculturalmente para o Brasil.

Devido ao baixo número de casos clínicos conhecidos atualmente, sugere-se que a equipe multiprofissional faça reuniões de acordo com a necessidade e o volume que o registro apresente. Mediante a documentação do paciente, ela irá avaliar os casos de mesotelioma, conforme a classificação proposta pelo registro da Clinica del Lavoro de Milão e, depois, registrá-los no banco de dados do software Renam (https://www.inail.it/cs/internet/attivita/ricerca-e-tecnologia/area-salute-sul-lavoro/ sorveglianza-epidemiologica-negli-ambienti-di-lavoro-e-di-vita/renam.html), que foi traduzido e adaptado para o Brasil em 2017 e que está disponível gratuitamente para download.

Com essa sistemática, é possível replicar no Brasil, guardadas as especificidades e as características do país, os procedimentos realizados na Itália, que têm se mostrado eficientes, adequados e satisfatórios, tanto do ponto de vista legal quanto de armazenamento, confidencialidade e gerenciamento de dados.

\section{Discussão}

Estudos 10,11,17,18,21 demonstram que a qualidade de dados dos RBP e RHC no Brasil são frágeis, devido a incompletude, duplicações ou inexistência de informações. No estudo 22 que avaliou a qualidade e a completude de dados enviados dos RHC para o IntegradorRHC, as variáveis estadiamento e grau de instrução tinham completude ruim. Para ocupação e origem do tratamento, a completude era regular. Outro estudo 17 que caracterizou a população com neoplasia, realizado no Estado do Rio de Janeiro, mostrou que $45 \%$ das informações relacionadas à ocupação eram incompletas ou inexistentes.

Nos resultados da fase exploratória da presente pesquisa, foi possível observar ausência de informações relacionadas a história ocupacional, discrepâncias para disponibilização e identificação de casos (CID ou morfologias), períodos de análise com tempos de coleta diferentes e casos duplicados em um mesmo registro. Essas situações dificultam, e muitas vezes impossibilitam, a avaliação e o conhecimento dos números reais de mesotelioma maligno e sua relação com o amianto. O estudo 22 que analisou a qualidade de informações, com verificação da completude e consistência das bases de dados, destacou que o problema da multiplicidade de casos dos RHC para o IntegradorRHC persiste. Isso é devido à diferença de tempo entre as bases estaduais e nacionais, dificultando a avaliação e o conhecimento da real veracidade das informações.

Quando se trata da variável ocupação, observou-se que em quase nenhuma base de dados é possível fazer a relação da atividade laboral e o surgimento da patologia, impossibilitando e dificultando a descoberta do nexo causal. Ao coletar as informações da história ocupacional, são encontrados dados da atividade atual ou, no máximo, da anterior, o que dificulta o conhecimento real da exposição ao amianto. Ainda que o preenchimento da variável ocupação nos RHC seja obrigatório, no estudo 18 que quantificou o percentual de taxa de ocupação nos RHC do Brasil, a ausência dessa informação para todas as topografias de câncer foi de $46 \%$. 
A presença de discrepâncias entre as bases de dados, bem como a falta da relação da ocupação com o uso do amianto, são comuns em países da América Latina, especificamente naqueles que não o baniram totalmente. No estudo 23 sobre a estimativa de mesotelioma maligno relacionado ao trabalho no Brasil, Argentina, Colômbia e México, apesar das diferenças econômicas entre os países, os dados relacionados à concentração de amianto são insignificantes ou quase inexistentes. Provavelmente isso decorre da inexistência de bancos de dados qualificados em algumas regiões ou de dados que não passam por controle de qualidade. $\mathrm{O}$ estudo ressalta a necessidade de melhoria dos registros nos quatro países.

$\mathrm{Na}$ direção oposta, países que têm se dedicado a pesquisar, discutir e registrar os casos de mesotelioma maligno conseguem apresentar dados consistentes, que permitem estimar mortalidade, relacionar o nexo causal com algumas ocupações e apresentar outras formas de contaminação como as exposições extraocupacional e ambiental. Estudos epidemiológicos são também possíveis de serem realizados para demonstrar o impacto negativo que o amianto gerou para a saúde da população.

Com base nos registros da Suécia, foi possível realizar um estudo de coorte entre 1961 e 2009 com 3.716 casos analisados. Desses, 785 (21,1\%) eram mulheres, 89,7\% tinham confirmação histológica para mesotelioma de pleura e em 24 atividades ocupacionais havia um risco aumentado para o desenvolvimento de mesotelioma 24 . A Austrália fez um investimento significativo na busca ativa de dados de pacientes diagnosticados com mesotelioma e exposição ao amianto 25 , realizando estimativas de mortalidade, identificando fontes de contágio e perspectivas futuras sobre novas formas de contaminação por amianto 26.

Nos Estados Unidos, em publicação de 2017 sobre a mortalidade do mesotelioma maligno 27, o número de casos de morte entre o período de 1999 a 2015 foi de 45.221, com aumento de 2.479 em 1999 para 2.597 em 2015. Os dados de óbitos com relação ocupacional somaram 96,3\% dos casos, com base em residentes de 23 estados norte-americanos, nos anos de 1999, 2003, 2004 e 2007.

Uma sensibilização importante no Brasil deve acontecer nos RHC e RCBP sobre a importância do abastecimento de dados para posterior envio ao IntegradorRHC, devido ao sistema ser uma das fontes de informação que possibilitam a avaliação da qualidade da atenção ao paciente oncológico. Entretanto, em se tratando de mesotelioma, registros específicos devem ser organizados para possibilitar o acompanhamento preciso dos casos, assim como acontece em outros países, com destaque para a Itália.

O Renam da Itália é um dos maiores sistemas de vigilância em epidemiologia do mundo 28 . A partir da sua implantação, foi possível observar melhoria, aumento e qualidade no fluxo de entrada de informações, cálculos da incidência dos casos com maior precisão e estabelecimento do nexo causal. Também houve possibilidade de realizar projeções futuras quanto ao número de casos, identificando e conhecendo novas fontes de exposição e contaminação 29.

No momento em que o registro italiano se tornou obrigatório, as instituições foram se comprometendo com a notificação. No primeiro relatório de dados do registro da região da Lombardia, 95,3\% das informações estavam disponíveis. O número de casos entre 2000 e 2004 foi de 1563, com documentação completa, em um total de 1957 casos suspeitos de mesotelioma maligno. Desse total, 573 casos foram confirmados como mesotelioma maligno, com nexo causal em 70,5\% e exposição certa ao amianto em $51,7 \%$ dos casos 30 .

Na segunda análise do registro da Lombardia, entre 2000 e 2008, o número de casos foi de 2.8161. Na terceira, entre 2000 e 2012, chegou-se a 4.442 casos, dos quais 60,9\% tiveram exposição ocupacional ao amianto 32 . O registro também possibilitou identificar a elevação do número de ocorrências por ano, com 277 casos em 2000 e um aumento de 68,7\% em 2012. A projeção para 2029 é de atingir 11 mil casos registrados 32 .

Merece destaque, no registro da Lombardia, a identificação de novas fontes de exposição e contaminação. No período de 2000 a 2002, 79 casos de mesotelioma maligno foram provenientes de indústria têxtil, pois nas máquinas de costura os sistemas de frenagem eram de amianto, expondo pessoas que os manipulavam ou faziam sua manutenção 33. Seis pacientes com mesotelioma maligno que realizaram atividades no serviço militar faziam manutenção de carros, tanques de guerra, ou participavam de brigadas de incêndio, manipulando produtos ou roupas produzidas com amianto 34 Quatro outros casos de trabalhadores do setor de entretenimento (cantor de ópera, ator, pessoal administrativo e de segurança) tiveram origem em ambientes com materias que continham amianto, como cortinas e paredes com isolante térmico 35 . 
A identificação da exposição, das fontes de contaminação e da relação com o amianto deve ser constantemente avaliada e verificada. Atualmente há uma grande quantidade de reservas naturais desse minério, existente em quarenta países, dos quais a extração não é proibida em 24 deles. Até 2017, 95\% da produção mundial estava concentrada em Rússia, Canadá, Brasil, Cazaquistão, China, Zimbábue e África do Sul 16.

No Brasil, em 2017, existiam 17 fábricas que usavam o amianto, localizadas em dez estados, sendo três no Paraná, nas cidades de Curitiba, Colombo e São José dos Pinhais 36. Segundo o Departamento Nacional de Produção Mineral, em 2013, o Paraná estava em primeiro lugar como o estado que mais consumiu amianto, o que justifica ser uma região propícia para iniciar um registro específico de casos de mesotelioma 16.

A proposta para implantação de um Registro de Casos de Mesotelioma em Curitiba pode ser embasada em três eixos principais: dificuldade ao acesso de dados exatos sobre número dos casos, proximidade de três grandes empresas que usavam o amianto e disposições legais estabelecidas na Lei Municipal no 14.172 15. Essa proposta poderá ser um piloto para futura implantação em outras localidades brasileiras.

Em relação aos dados sobre casos de mesotelioma, autores 21 destacam que no Brasil os dados ainda são limitados e sua qualidade não foi verificada. Assim, qualquer estimativa de taxas de incidência de mesoteliomas e câncer pleural e suas tendências é pouco mais do que uma suposição. Com exceção da cidade de São Paulo, os registros brasileiros de câncer se sobrepõem apenas de forma limitada às áreas geográficas caracterizadas por atividades industriais relacionadas ao amianto, o que dificulta o conhecimento real dos casos de mesotelioma no país. Dessa forma, a implantação de um registro de casos de mesotelioma em Curitiba apresenta-se como uma experiência a ser testada para posterior implantação em outras regiões do país.

Um grande avanço para todo território brasileiro foi a proibição da produção e comercialização do amianto tipo crisotila, que aconteceu em novembro de 2017. Anteriormente, alguns estados já tinham decidido por essa proibição, porém, em casos como o Paraná, ele era proibido somente em algumas cidades, sendo Curitiba um exemplo. Um próximo passo no Brasil seria a obrigatoriedade legal de criação de registros, seguindo o exemplo de muitos países nos quais a legislação os tornou obrigatórios. Em Curitiba, o Art. 6 da Lei Municipal no 14.17215 deixa clara a necessidade de programas de vigilância em saúde, quando destaca que "o SUS municipal de Curitiba, através dos serviços de Vigilância em Saúde, Centros de Referência em Saúde do Trabalhador e demais unidades da rede, é o responsável pelos programas de vigilância em saúde (...) ações que visem à prevenção (...) das doenças decorrentes do trabalho com amianto".

O parágrafo primeiro do mesmo artigo também destaca a obrigatoriedade da notificação dos casos de doenças relacionadas ao amianto quando diz: "Fica instituída a Notificação Obrigatória à autoridade local do SUS, pela rede pública e privada de assistência à saúde, de todos os casos e de doenças e óbitos decorrentes da exposição ao amianto". Também o parágrafo $3^{\circ}$ aborda a necessidade de comunicação: "Os casos de doenças é óbitos decorrentes da exposição ao amianto de origem ocupacional deverão ser encaminhados, via CAT [Comunicado de Acidente de Trabalho] ao INSS [Instituto Nacional de Seguro Social]" 15.

A previsão do pico do número de casos de mesotelioma no Brasil justifica a preocupação com o conhecimento da quantidade real dos casos. O país possivelmente terá até 203012 um aumento do número de casos. Dessa forma, a organização de registros com dados fidedignos sobre o número real de casos de mesotelioma maligno e a possibilidade de estabelecimento do nexo causal auxiliarão no conhecimento do impacto que o amianto causou no país.

\section{Conclusão}

A criação de um sistema de monitoramento dos casos de mesotelioma maligno com a implantação de um registro específico voltado a pacientes diagnosticados com a doença poderá ser um piloto na cidade de Curitiba, e posteriormente pode contribuir para a replicação de novos registros no Brasil. Com o aumento do número de casos previstos para as próximas décadas e o atendimento às demandas legais, esses registros tornam-se essenciais, a fim de, além de atenderem à legislação vigente, auxiliarem no acompanhamento, registro, determinação de nexo causal e fontes de contaminação específicas no país. 
Ao utilizar-se como modelo o caso italiano, reconhecido internacionalmente como bem-sucedido, pode-se disponibilizar gratuitamente os documentos traduzidos e adaptados para a realidade brasileira, de modo a criar modelos de registros que estejam adaptados e adequados ao nosso país. A criação desses registros nos principais centros do Brasil contribuirá, tal como aconteceu no caso italiano, para o acompanhamento e melhoria dos serviços de saúde prestados aos trabalhadores acometidos por essa moléstia.

\section{Colaboradores}

L. P. Kalinke contribuiu na concepção, projeto, análise e interpretação dos dados, redação do artigo e aprovação final da versão a ser publicada. M. A. Kalinke contribuiu na concepção, projeto e revisão crítica relevante do conteúdo intelectual. L. M. M. Sarquis colaborou na concepção, projeto e aprovação final da versão a ser publicada. L. Marcondes e T. Halfeld contribuíram no projeto, análise e interpretação dos dados e redação do artigo. C. Mensi e S. Consonni colaboraram na concepção, projeto, análise e interpretação dos dados e aprovação final da versão a ser publicada.

\section{Agradecimentos}

Coordenação de Aperfeiçoamento de Pessoal de Nível Superior (Capes), por meio do edital Professor Visitante Exterior, auxílio no 189571.

\section{Referências}

1. Marinaccio A, Binazzi A, Cauzillo G, Cavone D, Zotti RD, Ferrante P, et al. Analysis of latency time and its determinants in asbestos related malignant mesothelioma cases of the Italian register. Eur J Cancer 2007; 43:2722-8.

2. Rodriguez D, Cheung MC, Housri N, Koniaris LG. Malignant abdominal mesothelioma: defining the role of surgery. J Surg Oncol 2009; 99:51-5.

3. Branco BPC, Carvalho FA, Paiva FA, Branco ITC. Dificuldades no diagnóstico diferencial entre mesotelioma pleural e adenocarcinoma metastático para pleura patológica. J Bras Med 2013; 101:21-5.

4. Robinson BR, Musk AW, Lake RA. Malignant mesothelioma. Lancet 2005, 366:397-408.

5. Tian L, Zeng R, Wang X, Shen C, Lai Y, Wang $\mathrm{M}$, et al. Prognostic significance of soluble mesothelin in malignant pleural mesothelioma: a meta-analysis. Oncotarget 2017; 8:46425-35.

6. GBD 2015 Risk Factors Collaborators. Global, regional, and national comparative risk assessment of 79 behavioural, environmental and occupational, and metabolic risks or clusters of risks, 1990-2015: a systematic analysis for the Global Burden of Disease Study 2015. Lancet 2016 ; 388:1659-1724.

7. IARC Working Group on the Evaluation of Carcinogenic Risks to Humans. A review of human carcinogens. Part C: arsenic, metals, fibres, and dusts. Lyon: International Agency for Research on Cancer; 2009.

8. Salzgeber DC, Silva BS, Giannasi F. Denominação, produção e consumo no Brasil. Trabalho \& Saúde 2017; 42(Ed Especial).

9. Mensi C, Riboldi L, De Matteis S, Bertazzi PA, Consonni D. Impact of an asbestos cement factory on mesothelioma incidence: global assessment of effects of occupational, familial, and environmental exposure. Environ Int 2015; 74:191-9.

10. Terra Filho M, Freitas JBP, Nery LE. Doenças asbesto-relacionadas. J Bras Pneumol 2006; 32 Suppl 2:S48-53. 
11. World Health Organization. Asbestos: elimination of asbestos-related diseases. http:// www.who.int/mediacentre/factsheets/fs343/ en/ (acessado em 16/Ago/2017).

12. Algranti E, Saito CA, Carneiro APS, Moreira B, Mendonça EMC, Bussacos MA. The next mesothelioma wave: mortality trends and forecast to 2030 in Brazil. Cancer Epidemiol 2015; 39:687-92.

13. Instituto Nacional de Câncer José Alencar Gomes da Silva. Diretrizes nacionais para a vigilância do câncer relacionado ao trabalho. http://bvsms.saude.gov.br/bvs/publicacoes/ inca/diretrizes_vigilancia_cancer_trabalho. pdf (acessado em 13/Fev/2017).

14. Brasil. Lei Federal no 9.055, de 1o de junho de 1995. Disciplina a extração, industrialização, utilização, comercialização e transporte do asbesto/amianto e dos produtos que o contenham, bem como das fibras naturais e artificiais, de qualquer origem, utilizadas para o mesmo fim e dá outras providências. Diário Oficial da União 1995; 2 jun.

15. Câmara Municipal de Curitiba. Lei no 14.172 , de 07 de dezembro de 2012. Dispõe sobre a proibição do uso, no município de Curitiba, de materiais ou artefatos que contenham quaisquer tipos de amianto ou asbesto. Diário Oficial do Município 2012; $11 \mathrm{dez}$.

16. Departamento Nacional de Produção Mineral. Crisotila-amianto. Sumário mineral. http:// www.dnpm.gov.br/dnpm/sumarios/crisotilaamianto-sumario-mineral-2014/view (acessado em 13/Abr/2017)

17. Tabalipa MM, Boccolini PMM, Simões DR Chrisman JR, Otero UB, Garbin HBR, et al. Information on occupation in hospital cancer records in Rio de Janeiro State. Cad Saúde Colet (Rio J.) 2011; 19:278-86.

18. Grabois MF, Souza MC, Guimaraes RM, Otero UB. Completude da informação "Ocupação" nos Registros Hospitalares de Câncer do Brasil: bases para a vigilância do câncer relacionado ao trabalho. Rev Bras Cancerol 2014; 60:207-14.

19. Koller FJ, Sarquis LMM, Mantovani MF, Miranda FMA, Consonni D, Mensi C. Monitoramento do mesotelioma no sul do Brasil: uma realidade ainda a ser estudada. Cogitare Enferm 2017; 22:1-6.

20. Nesti M, Marinaccio A, Chellini E. La sorveglianza dei casi di mesotelioma maligno e la definizione delle esposizioni ad amianto: i dati ReNaM 1997. Epidemiol Prev 2003; 27:14753.

21. Terracini B, Pedra F, Ubirani O. Asbestos-related cancers in Brazil. Cad Saúde Pública 2015; 31:903-5.

22. Pinto IV, Ramos DN, Costa MCE, Ferreira CBT, Rebelo MS. Completude e consistência dos dados dos registros hospitalares de câncer no Brasil. Cad Saúde Colet (Rio J.) 2012; 20:113-20.
23. Pasetto R, Terracini B, Marsili D, Comba P. Occupational burden of asbestos-related cancer in Argentina, Brazil, Colombia, and Mexico. Ann Glob Health 2014; 80:263-8.

24. Plato N, Martinsen JI, Sparén P, Hillerdal G, Weiderpass E. Occupation and mesothelioma in Sweden: updated incidence in men and women in the 27 years after the asbestos ban. Epidemiol Health 2016; 38:e2016039.

25. Soeberg MJ, Leigh J, Zandwijk N. Malignant mesothelioma in Australia 2015: current incidence and asbestos exposure trends. J Toxicol Environ Health B Crit Rev 2016; 19:173-89.

26. Armstrong B, Driscoll T. Mesothelioma in Australia: cresting the third wave. Public Health Res Pract 2016; 26:e2621614.

27. Mazurek J, Syamlal G, Wood JM, Hendricks SA, Weston A. Malignant mesothelioma mortality - United States, 1999-2015. MMWR Morb Mortal Wkly Rep 2017; 66:214-8.

28. Mirabelli D, Cavone D, Merler E, Gennaro V, Romanelli A, Mensi C, et al. Non occupational exposure to asbestos and malignant mesothelioma in the Itálian National Registry of Mesotheliomas. Occup Environ Med 2010; 67: 792-94.

29. Mensi C, Termine L, Canti Z, Rivolta G, Riboldi L, Pesatori AC, et al. Il Registro mesoteliomi Lombardia, Centro Operativo Regionale (COR) del Registro nazionale mesoteliomi: aspetti organizzativi. Epidemiol Prev 2007; 31:283-9.

30. Nesti M, Marinaccio A, Cauzillo G, De Zotti R, Gennaro V, Gorini G, et al. I casi di mesotelioma maligno in Itália. Attività e risultati del Registro Nazionale (ReNaM). European Journal of Oncology 2005; 10:191-8.

31. Mensi C, Bonzini M, Macchione M, Sieno C, Riboldi L, Pesatori AC. Differences among peritoneal and pleural mesothelioma: data from the lombardy region mesothelioma register (Italy). Med Lav 2011; 102:409-16.

32. Mensi C, De Matteis S, Dallari B, Riboldi L, Bertazzi PA, Consonni D. Incidence of mesothelioma in Lombardy, Italy: exposure to asbestos, time patterns and future projections. Occup Environ Med 2016; 73:607-13.

33. Chiappino G, Mensi C, Riboldi L, Rivolta G. Il rischio amianto nel settore tessile: Indicazioni dal Registro Mesoteliomi Lombardia e definitiva conferma. Med Lav 2003; 94:521-30.

34. Canti Z, Mensi C, Riboldi L, Chiappino G. La denuncia di malattie causate dall'esposizione a rischi specifici durante il servizio militare. Med Lav 2005; 96:88-9.

35. Mensi C, Garberi A, Bordini L, Sieno C, Riboldi L. Asbestos-related diseases in entertainment workers. Med Lav 2010; 101:416-8.

36. Pedra F, Silva PO, Mattos IE, Castro HA. Mesothelioma mortality rate in Brazil, 1980 to 2010. Rev Bras Cancerol 2014; 60:199-206. 


\section{Abstract}

The study proposes the creation of a system to monitor cases of malignant mesothelioma in the municipality of Curitiba, Paraná State, Brazil, based on the Italian model. This diagnosis-type action-research project featured exploratory and planning phases conducted from July 2015 to May 2017. The following search tools were used: Hospital-Based Cancer Registries Integrator with specific morphologies for mesothelioma; HospitalBased Cancer Registry with codes C38.4 and C45 of the International Classification of Diseases, $10^{\text {th }}$ revision, and/or records coded by the ICD$O$ with topographies C38 and C48; PopulationBased Cancer Registry of the Curitiba Municipal Health Department, with the same codes. The study also identified, analyzed, and adapted to the Brazilian reality the model, questionnaires, and registry software for mesothelioma from Lombardy, Italy. Fifteen cases of mesothelioma were recorded in the Hospital-Based Cancer Registries Integrator. Two cases were recorded in the University Hospital-Based Cancer Registry and 16 in the Cancer Hospital. There were 317 cases recorded in the Population-Based Cancer Registry during the same period. Although some information was complete, data were lacking on patients' occupational history, thereby preventing the determination of a causal nexus. Given a predicted increase in cases of mesothelioma in the coming decades and the response to court cases, the implementation of registries has become essential to facilitate knowledge and follow-up on the determination of the causal link and specific sources of asbestos exposure in the country.

Mesothelioma; Asbestos; Health Information Systems; Information Management

\section{Resumen}

El objetivo de este estudio fue proponer la creación de un sistema de monitoreo de casos de mesotelioma maligno en el municipio de Curitiba, Paraná, Brasil, desarrollado en base al modelo italiano. Se trata de una investigación-acción de tipo diagnóstica, que usa fases de carácter exploratorio y de planificación, desarrolladas durante el período de julio de 2015 a mayo de 2017. Se utilizaron como herramientas de búsqueda los siguientes instrumentos: Integrador del Registro Hospitalario de Cáncer con morfologías específicas para mesotelioma; Registro Hospitalario de Cáncer con los códigos C38.4 y C45, procedentes de la Clasificación Internacional de Enfermedades décima revisión, $y /$ registros codificados por el CID-O con topografías C38 y C48; Registro de Cáncer de base poblacional de la Secretaría Municipal de Salud de Curitiba con los mismos códigos. También se presentaron, analizaron y adaptaron a la realidad brasileña el modelo, los cuestionarios y el software de registro de mesotelioma de Lombardía, en Italia. Se observó que, en el Integrador de Registro Hospitalario de Cáncer, se registraron $15 \mathrm{ca}$ sos de mesotelioma. En el Registro Hospitalario de Cáncer del hospital universitario, fueron dos. En el hospital oncológico, 16. Los datos del Registro de Cáncer de Base Poblacional, a su vez, indicaron 317 registros relacionados con este período. A pesar de que algunos datos estaban más completos, existe una falta de datos relacionados con la historia laboral, imposibilitando establecer el nexo causal. Con el aumento del número de casos de mesotelioma previstos para las próximas décadas, y la atención a las demandas legales, la implantación de los registros se convierte en esencial para apoyar el conocimiento, el seguimiento, así como la determinación del nexo causal y las fuentes de contaminación específicas en el país.

Mesotelioma; Asbestos; Sistemas de Información en Salud; Gestión de la Información
Recebido em 02/Out/2017

Versão final reapresentada em 16/Mar/2018 Aprovado em 03/Mai/2018 